\title{
Determination of the properties of the central engine in microlensed QSOs
}

\author{
L. J. Goicoechea ${ }^{1}$, D. Alcalde ${ }^{2}$, E. Mediavilla ${ }^{2}$, and J. A. Muñoz ${ }^{2}$ \\ 1 Departamento de Física Moderna, Universidad de Cantabria, Avda. de Los Castros s/n, 39005 Santander, Spain \\ e-mail: goicol@unican.es \\ 2 Instituto de Astrofísica de Canarias, C/ Vía Láctea s/n, 38200 La Laguna, Spain \\ e-mail: dalcalde@ll.iac.es; emg@ll.iac.es; jmunoz@ll.iac.es
}

Received 17 July 2002 / Accepted 7 October 2002

\begin{abstract}
We study a recently observed gravitational microlensing peak in the $V$-band light curve of Q2237+0305A using a relatively simple physical model, but one that is highly consistent with the data (the best-fit reduced $\chi^{2}$ is very close to 1 ). The source quasar is assumed to be a Newtonian geometrically-thin and optically-thick accretion disk. The disk has an arbitrary orientation, and both blackbody and greybody emission spectra are considered. When the electron-photon scattering plays a role, the greybody spectrum will be a simplified version of the exact one. In our model, the microlensing variability results from the source crossing a caustic straight line. The main goal of our work is to estimate the black hole mass and the mass accretion rate in QSO 2237+0305 as well as to discuss the power and the weakness of the technique, some possible improvements, and future prospects from multifrequency monitoring of new microlensing peaks. We also put into perspective the new methodology and the results on the central engine in QSO 2237+0305. From the fitted microlensing parameters and reasonable dynamical/cosmological constraints, it is concluded that QSO 2237+0305 harbours a central massive black hole: $10^{7} M_{\odot}<M<6 \times 10^{8} M_{\odot}$. While the information about the central dark mass is very interesting, the mass accretion rate is not so well constrained. The typical values of the disk luminosity/Eddington luminosity ratio are in the $(1-20) \varepsilon$ range, where $\varepsilon \leq 1$ is the emissivity relative to a blackbody and the highest $L / L_{\mathrm{Edd}}$ ratio corresponds to the largest deflector motion. Therefore, in order to verify $L / L_{\text {Edd }} \leq 1$, a relatively small projected peculiar motion of the lens galaxy and a greybody emission seem to be favored.
\end{abstract}

Key words. gravitational lensing - galaxies: nuclei - quasars: general - quasars: individual: Q2237+0305

\section{Introduction}

The optical continuum from a QSO could originate from a geometrically-thin and optically-thick standard accretion disk. This standard scenario is able to explain most of the nonvariable background component, whereas the fluctuations on different time scales may be caused by different mechanisms (accretion disk instabilities, supernova explosions in a circumnuclear stellar region, and so on). The Newtonian model of a standard accretion disk around a black hole was introduced by Shakura \& Sunyaev (1973). In this model, the released gravitational energy is emitted as a multitemperature blackbody radiation, where $T_{\mathrm{s}}(r)$ is the temperature at radius $r$. Thus, in the absence of extinction, the emitted and observed intensities obey Planck laws $B_{v_{\mathrm{s}}}\left[T_{\mathrm{s}}(r)\right]$ and $B_{\gamma}[T(r)]$, respectively, where the emitted temperature and the observed one are related through the cosmological redshift of the source $z_{\mathrm{s}}: T(r)=T_{\mathrm{s}}(r) /\left(1+z_{\mathrm{s}}\right)$. As the emitted temperature profile depends on the black hole mass and the mass accretion rate, the actually observed intensity profile $I_{v}(r)=\epsilon_{v} B_{v}[T(r)]$ is determined from the two physical parameters of the source. In a general situation we must

Send offprint requests to: L. J. Goicoechea,

e-mail: goicol@unican.es take into account an extinction factor $\epsilon_{v}$ due to dust in the host galaxy of the quasar and any galaxy in the line of sight including a possible lens galaxy and the Milky Way. A relativistic version of the Shakura \& Sunyaev model was presented by Novikov \& Thorne (1973) and Page \& Thorne (1974). The relativistic model should be a useful tool to describe the physics of the innermost layers of the disk. However, as we are interested in the emissivity of a wide optical source from an inner edge of several Schwarzschild radii up to an outer edge of $10^{2}-10^{3}$ Schwarzschild radii, to a first approximation, we can ignore the relativistic effects and take the Newtonian picture.

When a lensed quasar crosses a microcaustic, an important fluctuation in the flux of one of its images is seen, i.e., a gravitational microlensing high-magnification event (HME) appears. The microlensing light curve of the image involved is given by convolving the observed intensity distribution with the corresponding magnification pattern. Therefore, the behaviour of the HME depends on the physical properties of the source quasar, and this fact opens discussion on the structure of the source from the analysis of the prominent microlensing event (e.g., Rauch \& Blandford 1991; Jaroszyński et al. 1992). In principle, if we assume a standard accretion disk as the source of the optical continuum, a measurement of the black hole mass and 
the mass accretion rate may be possible and, recently, several papers have indicated that the study of an individual HME may be used to infer these parameters (Yonehara et al. 1998; Agol \& Krolik 1999; Mineshige \& Yonehara 1999; Yonehara et al. 1999; Shalyapin 2001; Yonehara 2001).

Very recently, the GLITP (Gravitational Lenses International Time Project) collaboration has monitored the four images of QSO $2237+0305$ in the $V$ and $R$ bands. In each optical band, the GLITP light curve for the brightest image, Q2237+0305A, traced the peak of one HME with unprecedented quality (Alcalde et al. 2002). The global flat shape for the light curve of the faintest image, Q2237+0305D, suggested that the intrinsic signal is globally stationary, and this result supported the contention that the global variability in Q2237+0305A is exclusively caused by microlensing. The peak of the HME was fitted to the microlensing curves resulting from face-on circularly-symmetric sources crossing a single straight fold caustic, and it was determined that the only source models totally consistent with the GLITP data are the Newtonian standard accretion disk and its simplified versions (Shalyapin et al. 2002). The uniform and Gaussian disks led to an excessively high value of the reduced chi-square, but the simplified variants of the Newtonian standard accretion disk gave excellent results: the best fits are in very good agreement with the observations and the upper limits on the source size are highly reasonable. To avoid a possible anomalous ratio of fitted background fluxes $F_{0 R} / F_{0 V}$, one needs to consider additional $R$ light, which is emitted from an extended region (e.g., Jaroszyński et al. 1992). This possible $R$-band extended source is however irrelevant in the estimation of the compact source size as well as in the measurements of the central mass and the accretion rate from the technique presented in this paper. Unfortunately, from the framework used by Shalyapin et al. (2002), we cannot measure the physical parameters of the engine. One can only determine a relationship between the black hole mass $(M)$, the mass accretion rate $(\mathrm{d} M / \mathrm{d} t)$, and the quasar velocity perpendicular to the caustic line $\left(V_{\perp}\right)$. Even from some reasonable velocity range, there is no way to separately infer $M$ and $\mathrm{d} M / \mathrm{d} t$.

In this paper (Sect. 2), we introduce a novel expression of the microlensing light curve when a Newtonian standard source crosses a single straight fold caustic. This novel approach permits one to break the degeneracy in the estimation of $M$ and $\mathrm{d} M / \mathrm{d} t$, and so to measure these parameters for a source with an arbitary orientation. In Sect. 3, from the $V$-band microlensing peak found by the GLITP collaboration, we obtain estimates of the black hole mass and the mass accretion rate in QSO 2237+0305. In Sect. 4 we present the main conclusions and put into perspective the technique and the new results on the central engine in QSO 2237+0305.

\section{Microlensing light curve for a Newtonian standard accretion disk near a caustic straight line}

Yonehara et al. (1998) and Shalyapin et al. (2002) previously studied the microlensing light curve associated with a Newtonian standard source that crosses a single straight fold caustic. Yonehara et al. (1998) presented the expected light curve when a face-on source $\left(M=10^{8} M_{\odot}, \mathrm{d} M / \mathrm{d} t \approx\right.$ $0.13 M_{\odot} \mathrm{yr}^{-1}$ ) is strongly magnified by a given caustic line, while Shalyapin et al. (2002) discussed the time evolution of the monochromatic flux for a face-on source (the inner and outer edges were assumed to be $r_{\text {in }}=0$ and $r_{\text {out }}=\infty$, respectively) near to a generic caustic line. Using the theoretical light curve reported by Shalyapin et al. and an observed HME, one is able to obtain information on the size of the involved source quasar, provided that some interval of the quasar velocity $V_{\perp}$ might be inferred from observational data. However, their approach does not permit a measurement of the two main parameters of the source: the central mass and the mass accretion rate.

Here we show the behaviour of an HME caused by a generic Newtonian standard accretion disk (with finite inner and outer edges, and an arbitrary orientation) crossing a generic caustic straight line, and more importantly, a framework in which it is possible to measure the physical properties of the black hole-accretion disk complex. We begin with a basic ingredient of the monochromatic radiation flux: the observed intensity profile. The observed intensity from a part of the disk at $(r, \psi)$ is given by

$I_{v}(r)=\epsilon_{v} B_{v}[T(r)]=\frac{2 h v^{3}}{c^{2}} \frac{\epsilon_{v}}{\exp [h v / k T(r)]-1}$,

where (Shakura \& Sunyaev 1973)

$T(r)=\left[\frac{3}{8 \pi} \frac{G M}{\sigma r^{3}}\left(\frac{\mathrm{d} M}{\mathrm{~d} t}\right)\left(1-\sqrt{\frac{r_{\mathrm{in}}}{r}}\right)\right]^{1 / 4}\left(1+z_{\mathrm{s}}\right)^{-1}$.

The temperature profile depends on the black hole mass $(M)$ and the mass accretion rate $(\mathrm{d} M / \mathrm{d} t)$, as well as the redshift of the source $\left(z_{\mathrm{s}}\right)$ and the inner edge of the disk $\left(r_{\text {in }}\right)$. Two physical constants are also involved: the Stefan constant $(\sigma)$ and the gravitation constant $(G)$. As usual, $r_{\text {in }}$ is assumed to be three times the Schwarzschild radius of the black hole, i.e., $r_{\text {in }}=6 G M / c^{2}$. Both the temperature and the intensity are circularly symmetric. Another basic ingredient is the magnification pattern. In our problem (magnification near to a straight fold caustic), the magnification law is simple and well-known. Taking a Cartesian coordinate frame in which the caustic line is defined by the $y$-axis, the magnification of a piece of the disk at $(x, y)$ will be (e.g., Schneider \& Weiss 1987)

$A(x)=A_{0}+a_{\mathrm{C}} H(x) / \sqrt{x}$,

where $H(x)=1$ at $x>0$ and $H(x)=0$ at $x \leq 0$. In the new coordinate frame, the magnification law is a function of the coordinate $x$, and we can easily relate this Cartesian coordinate to the corresponding ones $(r, \psi)$ in the natural coordinate frame of the disk. If the centre of the source is placed at $x=x_{\mathrm{c}}$, we find a relationship between $x$ and $(r, \psi)$ :

$x=x_{\mathrm{c}}+r \sin \psi \cos \alpha \sin \beta-r \cos \psi \cos \beta$.

To derive Eq. (4) it was considered that the axis of the disk is inclined by an angle $\alpha$ in the plane of the observer's sky, and the node line and the $x$-axis are separated by an angle $\beta$. The elemental flux from the surface element $r \mathrm{~d} r \mathrm{~d} \psi$ can be 
now estimated in a direct way. In the absence of the lens, $\mathrm{d} \Omega_{*}=D_{\mathrm{s}}^{-2} r \mathrm{~d} r \mathrm{~d} \psi \cos \alpha$ is the solid angle subtended in the observer's sky by the piece of the disk, where $D_{\mathrm{s}}$ is the angular diameter distance to the source. The true solid angle will be $\mathrm{d} \Omega=$ $A\left[x\left(r, \psi ; x_{\mathrm{c}}\right)\right] \mathrm{d} \Omega_{*}$, and consequently, the elemental flux has an expression $\mathrm{d} F_{v}\left(r, \psi ; x_{\mathrm{c}}\right)=D_{\mathrm{s}}^{-2} I_{v}(r) A\left[x\left(r, \psi ; x_{\mathrm{c}}\right)\right] r \mathrm{~d} r \mathrm{~d} \psi \cos \alpha$. Moreover, the integral of $\mathrm{d} F_{v}\left(r, \psi ; x_{\mathrm{c}}\right)$ over the whole disk gives the total monochromatic flux $F_{v}\left(x_{\mathrm{c}}\right)$, i.e.,

$$
\begin{aligned}
F_{v}\left(x_{\mathrm{c}}\right)= & K_{v} \int_{r_{\text {in }}}^{r_{\text {out }}} \frac{r \mathrm{~d} r}{\exp [h v / k T(r)]-1} \\
& \times \int_{0}^{2 \pi}\left\{1+\frac{a_{\mathrm{C}}}{A_{0}} \frac{H\left[x\left(r, \psi ; x_{\mathrm{c}}\right)\right]}{\sqrt{x\left(r, \psi ; x_{\mathrm{c}}\right)}}\right\} \mathrm{d} \psi,
\end{aligned}
$$

where $K_{v}=\left(2 h v^{3} / c^{2} D_{\mathrm{s}}^{2}\right) A_{0} \epsilon_{v} \cos \alpha$, and $r_{\text {out }}$ is the outer edge of the disk, which $\left(r_{\text {out }}\right)$ could be of the order of 100-300 inner $\operatorname{radii}\left(r_{\text {in }}\right)$.

In order to derive the microlensing light curve from Eq. (5), we firstly calculated the integral over $\psi$. The angular integration led to

$\int_{0}^{2 \pi}\left[1+\frac{a_{\mathrm{C}}}{A_{0}} \frac{H(x)}{\sqrt{x}}\right] \mathrm{d} \psi=2 \pi\left[1+\frac{a_{\mathrm{C}}}{\pi A_{0} \sqrt{r f}} G(q)\right]$,

where $f=\left(\cos ^{2} \beta+\cos ^{2} \alpha \sin ^{2} \beta\right)^{1 / 2}, q=x_{\mathrm{c}} / r f$, and

$G(q)=\int_{-1}^{+1} \frac{H(q-y) \mathrm{d} y}{\sqrt{q-y} \sqrt{1-y^{2}}}$.

Inserting all these results into Eq. (5), one obtains

$$
\begin{aligned}
F_{v}\left(x_{\mathrm{c}}\right) & =2 \pi K_{v} \\
& \times \int_{r_{\text {in }}}^{r_{\text {out }}}\left[1+\frac{a_{\mathrm{C}}}{\pi A_{0} \sqrt{r f}} G\left(x_{\mathrm{c}} / r f\right)\right] \frac{r \mathrm{~d} r}{\exp [h v / k T(r)]-1} \cdot(8)
\end{aligned}
$$

In a second step, we used the new variable $\xi=r / r_{\text {in }}$ as well as the trajectory of the centre of the source $x_{\mathrm{c}}(t)=V_{\perp}\left(t-t_{0}\right)$. As usual, $V_{\perp}$ is the quasar velocity perpendicular to the caustic line, and $t_{0}$ is the time of caustic crossing by the source centre. The final light curve has an expression

$$
\begin{aligned}
F_{v}(t)= & A_{v} \int_{1}^{\xi_{\max }}\left\{1+\frac{B}{\sqrt{\xi}} G\left[C\left(t-t_{0}\right) / \xi\right]\right\} \\
& \times \frac{\xi \mathrm{d} \xi}{\exp \left[0.054\left(\frac{v}{10^{14} \mathrm{~Hz}}\right) D \xi^{3 / 4}(1-1 / \sqrt{\xi})^{-1 / 4}\right]-1},
\end{aligned}
$$

where

$$
\begin{aligned}
& A_{v}=\frac{2 \pi r_{\text {in }}^{2}}{D_{\mathrm{s}}^{2}}\left(\frac{2 h v^{3}}{c^{2}}\right) A_{0} \epsilon_{v} \cos \alpha, \\
& B=\frac{a_{\mathrm{C}}}{\pi A_{0}\left(r_{\mathrm{in}} f\right)^{1 / 2}}, \\
& C=\frac{V_{\perp}}{r_{\text {in }} f}, \\
& D=\left(\frac{M}{10^{8} M_{\odot}}\right)^{1 / 2}\left(\frac{\mathrm{d} M / \mathrm{d} t}{10^{26} \mathrm{~g} \mathrm{~s}^{-1}}\right)^{-1 / 4} .
\end{aligned}
$$

In Eq. (9), $\xi_{\max }$ is the ratio between the outer radius and the inner radius, i.e., $\xi_{\max }=r_{\text {out }} / r_{\text {in }}$. Moreover, in order to reduce the computing time, from some MATHEMATICA packages, we inferred an analytical approximation to the function $G(q)$ (see Appendix A). The exact function is very well traced by the approximated one, with a typical accuracy of about $0.01 \%$. We note that the time evolution of the theoretical HME depends on the frequency, two chromatic amplitudes $\left(A_{v}, B_{v}=B A_{v}\right)$, and four achromatic parameters $\left(\xi_{\max }, C, D\right.$, and $\left.t_{0}\right)$.

In practice one must compare the theoretical law (9) with the brightness record of a QSO image during a HME. In the comparison, the frequency $v$ will be the central frequency of the optical band in which the observations were made, and as mentioned above, $\xi_{\max }=100$ ( $r_{\text {out }}=300$ Schwarzschild radii) or $\xi_{\max }=300\left(r_{\text {out }} \sim 1000\right.$ Schwarzschild radii $)$ are plausible choices of the edge ratio. Therefore, at a given $\xi_{\max }$ (e.g., 100), the goal is to estimate the values of the parameters $A_{v}, B_{v}, C$, $D$, and $t_{0}$ by fitting the observed HME. Once the parameters $C$ and $D$ have been measured, from Eqs. (12)-(13) we make the relations:

$\mu_{\mathrm{BH}}=10^{-4} V_{\perp}\left(\mathrm{km} \mathrm{s}^{-1}\right) /\left[f C\left(\mathrm{day}^{-1}\right)\right]$

$\mu_{\mathrm{AC}}=\mu_{\mathrm{BH}}^{2} / D^{4}$

with $\mu_{\mathrm{BH}}=M / 10^{8} M_{\odot}$ and $\mu_{\mathrm{AC}}=(\mathrm{d} M / \mathrm{d} t) / 10^{26} \mathrm{~g} \mathrm{~s}^{-1}$. Although the factor related to the orientation of the source $(f)$ cannot be directly obtained from the fit, its estimation is relatively simple. We simulated $10^{4}$ pairs of values $(\alpha, \beta)$ covering the whole range of possible orientations, and calculated the corresponding $f$ values. From the simulations, we got and drew (Fig. 1) the probability distribution $P(f)$. In Fig. 1 it is apparent that the bulk of the probability is associated with high values of $f$, and the maximum of the distribution is reached when $f \rightarrow 1$. Only very particular orientations (nearly edge-on disks with a node line almost parallel to the caustic line) lead to small values of the factor $f$. From the probability distribution we inferred that $\langle f\rangle=0.84$ and $f=0.84_{-0.02}^{+0.16}$ at $1 \sigma$ confidence level (68.9 percent of the simulations; see the shaded region in Fig. 1). Finally, in order to measure the central mass and the flow of matter, only dynamical information is required. When that information is available, through Eqs. (14)-(15) one can estimate both physical quantities.

\section{Physical properties of the accretion disk in QSO 2237+0305}

The GLITP collaboration has recently reported information relating to the peak of a microlensing high-magnification event detected in both $V$-band and $R$-band brightness records of Q2237+0305A, which was observed with an excellent sampling rate of about three times the OGLE one (Alcalde et al. 2002). The event was discovered by the OGLE team (Woźniak et al. 2000), who followed the evolution of the whole $V$-band HME. In a first paper, from the GLITP $V$-band and $R$-band peaks, Shalyapin et al. (2002) have analyzed the nature and size of the $V$-band and $R$-band sources in QSO 2237+0305. To do this, they used a family of source models. All the models 


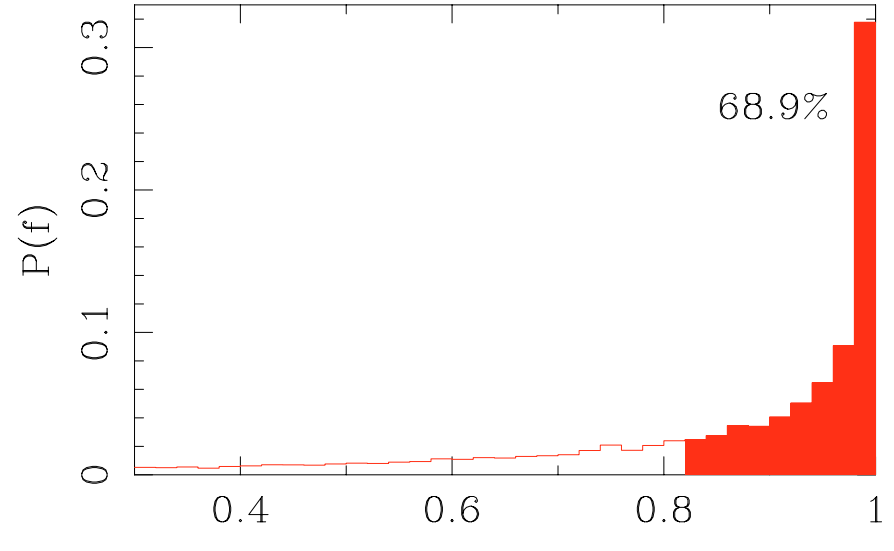

f

Fig. 1. Probability distribution of the factor related to the orientation of the source. The shading region represents the $1 \sigma$ confidence interval including both the mean value and the most probable one.

led to theoretical microlensing curves with the same number of free parameters. This approach (comparison between models causing four-parametric theoretical curves) is very useful for discussion of the feasibility of different scenarios. The authors concluded that only two rough versions of the Newtonian standard scenario are clearly consistent with the observed optical peaks. Thus we know that a Newtonian standard accretion disk is in agreement with the GLITP monitoring of Q2237+0305A, and in principle, it is possible to measure the central mass and the flow of matter (see Sect. 2).

\subsection{Measurements of $f C$ and $D$}

In our microlensing experiment, the functional relation presented in Eq. (9) is investigated by comparing it with the observed value of $F_{v}(t)$ at a given frequency and for various epochs $t_{1}, t_{2}, \ldots, t_{N}$. A value of $\xi_{\max }$ is also assumed. The goal is to find the parameters $A_{v}, B_{v}, C, D$, and $t_{0}$ of the theoretical microlensing curve which best describe the observational data. To make the fit, two different chi-square minimizations have been carried out. The most elementary procedure is to form a grid of points in the free parameters and evaluate the $\chi^{2}$ function at each of these points. The point with the smallest value is then the minimum. Therefore, we have studied a reduced grid using some MATHEMATICA packages. The procedure is called RGMath and it is based on the ideas discussed in Sect. 2.3 of Shalyapin et al. (2002). Basically, as we like to fit a theoretical curve that is linear in two parameters $\left(A_{v}\right.$ and $B_{v}$ ), and non-linear in $C, D$, and $t_{0}$, the equations of minimization $\partial \chi^{2} / \partial A_{v}=\partial \chi^{2} / \partial B_{v}=0$ lead to analytic relations: $A_{v}=A_{v}\left(C, D, t_{0}\right), B_{v}=B_{v}\left(C, D, t_{0}\right)$. In this scheme, the number of effective parameters is reduced to three, and one can work with a $3 \mathrm{D}$ grid instead of a 5D one. As the procedure is quite time consuming, we initially formed a 3D grid with moderate resolution, i.e., the size of the grid step was reasonable, but relatively large. So, from RGMath at moderate resolution, we inferred an initial best solution and some uncertainties associated with it. Another procedure is called DSFor and it is related to a version of the simplex method. We wrote a FORTRAN program to apply a downhill simplex methodology (Nelder \& Mead 1965) to our problem. The simplexes will be the simplest geometrical figures in 5 dimensions having 6 vertices (points). The technique begins by choosing a point (vertice) in the $5 \mathrm{D}$ parameter space. The program then generates an initial simplex, and after, repeated calculations are made while varying the vertices of the initial simplex in some way, until a local minimum is reached. The $\chi^{2}$ function may have different local minima around different points tested by the method, and consequently, to find the global minimum $\chi^{2}(\mathrm{~min})$ we must make a 5D grid, and apply the downhill simplex technique to all the points in the grid. In the first stage with this alternative task (search for minima in the 5D parameter space), the resolution was not very good. Once the initial best solution $\boldsymbol{v}^{*}=\left(A_{v}^{*}, B_{v}^{*}\right.$, $C^{*}, D^{*}, t_{0}^{*}$ ) is known (via RGMath or DSFor), it is possible to refine it and estimate accurate errors for the relevant parameters $C$ and $D$. If the vector $\boldsymbol{v}=\left(A_{v}, B_{v}, C, D, t_{0}\right)$ of parameter values is perturbed away from $\boldsymbol{v}^{*}$, then $\chi^{2}$ increases. So, we can draw high-resolution "parabolic" laws $\chi_{C}^{2}(\mathrm{~min})$ and $\chi_{D}^{2}(\mathrm{~min})$, where, for example, $\chi_{C}^{2}(\mathrm{~min})$ represents the minima of the $\chi^{2}$ function for values of $C$ around $C^{*}$. The $C$ range within which $\chi_{C}^{2}(\min )-\chi^{2}(\min ) \leq 1$ defines the $1 \sigma$ confidence interval in the estimation of $C$ and the interval $\chi_{D}^{2}(\min )-\chi^{2}(\min ) \leq 1$ is related to the $1 \sigma$ confidence interval in the estimation of $D$. The $k \sigma$ confidence interval in the generic parameter $p$ corresponds to the region bounded by $\chi_{p}^{2}(\min )-\chi^{2}(\min )=k^{2}(k=1,2, \ldots)$.

The high-resolution fit to the GLITP $V$-band light curve for $\mathrm{Q} 2237+0305 \mathrm{~A}$ appears in Table 1. The central frequency is $v_{V}=5.52 \times 10^{14} \mathrm{~Hz}$, and we adopted $\xi_{\max }=100$. We note that the uncertainties presented in Table 1 are $1 \sigma$ intervals. Moreover, instead of direct measurements of the parameter $C$, $1 \sigma$ confidence intervals for the relevant factor $f C$ (see Eq. (14)) are quoted in Table 1 . During an advanced stage of the project, we also obtained rough estimates of the errors in $A_{v}$ and $B_{v}$. From $D S F o r$ we inferred $A_{v} \sim 3_{-1}^{+6} \mu \mathrm{Jy}$ and $B_{v} \sim 1.5_{-0.6}^{+2.6} \mu \mathrm{Jy}$. In Fig. 2 we show the details to obtain the best values of $C$ and the standard errors. The figure presents three $\chi_{C}^{2}(\min )$ trends, which were derived from RGMath at moderate resolution (dashed line), RGMath at high resolution (solid line), and DSFor at high resolution (dotted line). The $1 \sigma$ box corresponding to the RGMath (high resolution) procedure is plotted with a thin line. Looking at both Table 1 and Fig. 2, two important conclusions appear: (1) the fit is stable against a change in the fitting method (RGMath or DSFor), and (2) a change from moderate to high resolution does not strongly perturb the parabolic law. Taking into account these last conclusions, we used the RGMath task (moderate resolution) to test the influence of the $\xi_{\max }$ value in the parameter estimation. We concluded that a change in $\xi_{\max }$ (from 100 to 300) does not modify the estimates of $f C$ and $D$. In principle, a fit to the GLITP $R$-band light curve for Q2237+0305A could be useful to improve the parameter estimation. However, the GLITP $R$-band microlensing peak does not permit one to infer the parameters $C$ and $D$ with errors similar to the previous ones (from the $V$-band peak). The ratio between the $1 \sigma R$-band interval and the $1 \sigma V$-band interval is $>2.4$ for $C$ and $\approx 1.5$ for $D$. Due to these relatively large errors from the $R$-band brightness record, 
Table 1. Parameters of the GLITP $V$-band microlensing peak.

\begin{tabular}{ccccccc}
\hline \hline Procedure & $\xi_{\max }$ & $A_{v}(\mu \mathrm{Jy})$ & $B_{v}(\mu \mathrm{Jy})$ & $f C\left(\right.$ day $\left.^{-1}\right)$ & $D$ & $t_{0}(\mathrm{JD}-2450000)$ \\
\hline RGMath & 100 & 2.78 & 1.37 & $0.23_{-0.06}^{+0.13}$ & $0.59_{-0.23}^{+0.30}$ & 1481.4 \\
DSFor & 100 & 2.96 & 1.35 & $0.23_{-0.08}^{+0.14}$ & $0.60_{-0.26}^{+0.35}$ & 1481.9 \\
\hline
\end{tabular}

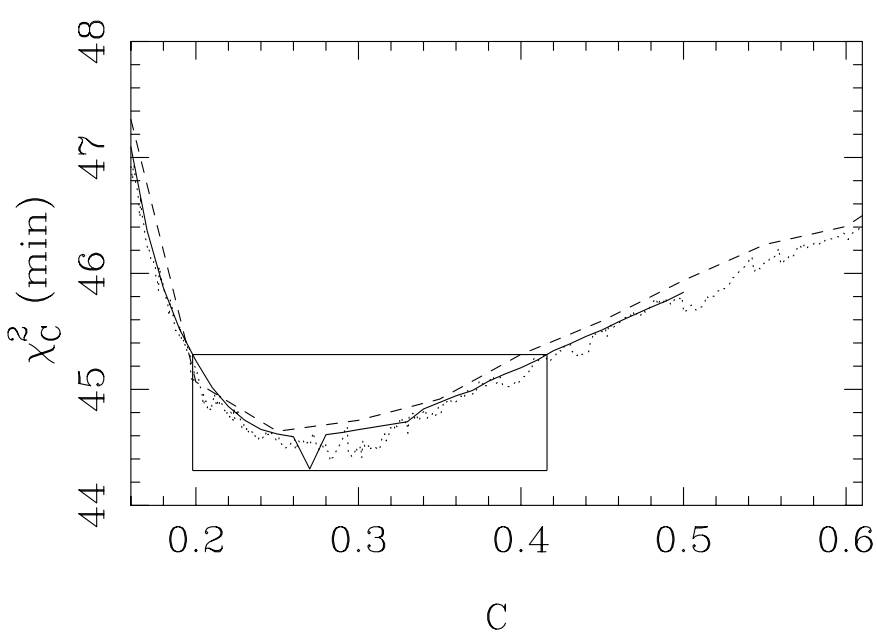

Fig. 2. Behaviour of $\chi_{C}^{2}(\min )$ derived from the GLITP $V$-band light curve for Q2237+0305A. The results with RGMath are shown by a dashed line (moderate resolution) and a solid line (high resolution). The dotted line was obtained with the DSFor task (high resolution). The $1 \sigma$ box corresponding to the RGMath procedure (high resolution) is also depicted.

we only considered the estimates in Table 1. More properly, we took $f C=0.23_{-0.06}^{+0.13} \mathrm{day}^{-1}$ and $D=0.59_{-0.23}^{+0.30}$ (RGMath solution) to determine the possible values of $\mu_{\mathrm{BH}}$ and $\mu_{\mathrm{AC}}$. We remark that the accretion-disk model is consistent with the $V$-band and $R$-band light curves. The best-fit reduced $\chi^{2}$ is very close to one in both optical filters, and we can see the good agreement between best-fits and observational trends in Fig. 3. In the top panel, the observed $V$-band fluxes are compared with the best-fit from RGMath at high resolution (solid line), while in the bottom panel, the $R$-band record is compared with the best-fit from RGMath at moderate resolution (dashed line). The fit in the $R$ band led to best values of $C=0.375$ day $^{-1}$ and $D=0.45$, which are totally consistent with the blue parameters given in Table 1 . However, the $R$-band data are relatively noisy in comparison to the $V$-band ones, and this fact does not permit a parameter estimation in the red band with uncertainties similar to the blue errors.

\subsection{Adoption of a dynamical range}

Kayser et al. (1986) showed that the effective transverse velocity of the source consists of three terms related to the transverse peculiar velocity of the source quasar $\left(\boldsymbol{v}_{\mathrm{s}}\right)$, the transverse peculiar velocity of the deflector $\left(\boldsymbol{v}_{\mathrm{d}}\right)$, and the transverse peculiar motion of the observer $\left(\boldsymbol{v}_{\mathrm{o}}\right)$. The word "transverse" denotes
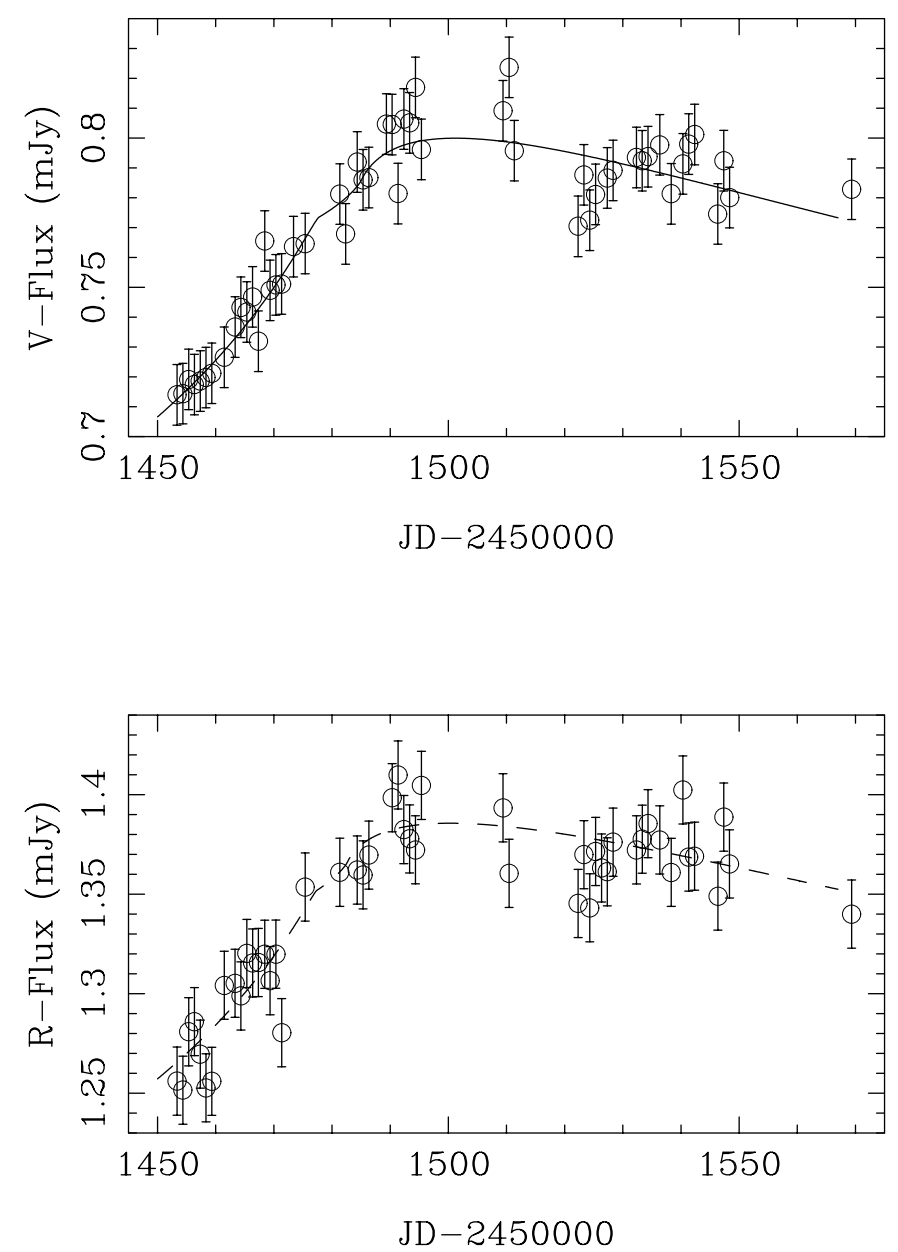

Fig. 3. Observed fluxes and best-fits. In the top panel ( $V$-band), it is showed the best-fit from RGMath at high resolution (solid line). In the bottom panel ( $R$-band), it appears the best-fit from RGMath at moderate resolution (dashed line).

"perpendicular to the line of sight", and the effective transverse velocity is given by

$\mathbf{V}=\frac{\boldsymbol{v}_{\mathrm{s}}}{1+z_{\mathrm{s}}}-\frac{\boldsymbol{v}_{\mathrm{d}}}{1+z_{\mathrm{d}}} \frac{D_{\mathrm{s}}}{D_{\mathrm{d}}}+\frac{\boldsymbol{v}_{\mathrm{o}}}{1+z_{\mathrm{d}}} \frac{D_{\mathrm{ds}}}{D_{\mathrm{d}}}$

where $z_{d}$ is the redshift of the deflector (lens galaxy), $D_{\mathrm{d}}$ is the angular diameter distance to the deflector, and $D_{\mathrm{ds}}$ is the angular diameter distance between the deflector and the source. In our case (QSO 2237+0305), the deflector is a nearby spiral galaxy $\left(z_{\mathrm{d}}=0.039<<1\right)$ and the source is a far quasar $\left(z_{\mathrm{s}}=\right.$ 1.695). Due to these facts, one has that $\left(1+z_{\mathrm{s}}\right) /\left(1+z_{\mathrm{d}}\right)=2.6$ and $D_{\mathrm{ds}} / D_{\mathrm{d}} \sim D_{\mathrm{s}} / D_{\mathrm{d}} \sim 10$, and the particular configuration permits one to neglect the term caused by the peculiar motion of the source. With respect to the motion of the observer, from the dipole observed in the cosmic microwave background (CMB), 
a peculiar velocity of the Sun is derived: $v_{\mathrm{obs}}=369 \mathrm{~km} \mathrm{~s}^{-1}$ in the direction $(\mathrm{J} 2000) \alpha=11^{\mathrm{h}} 11^{\mathrm{m}} 57^{\mathrm{s}}, \delta=-7.22$ (Lineweaver et al. 1996). This peculiar motion is almost perpendicular to the position for the system, $\left|\boldsymbol{v}_{\mathrm{o}}\right| \approx 50 \mathrm{~km} \mathrm{~s}^{-1}$. Because of the observer's small motion (compared to the expected transverse peculiar velocity of the nearby spiral), we can also neglect the last term in Eq. (16). Therefore, for QSO 2237+0305, the effective transverse velocity takes the very simple form

$\boldsymbol{V}=-\frac{\boldsymbol{v}_{\mathrm{d}}}{1+z_{\mathrm{d}}} \frac{D_{\mathrm{s}}}{D_{\mathrm{d}}}$

We are indeed interested in the effective quasar velocity perpendicular to the microcaustic of interest. Thus, if $i$ is the unit vector in the direction perpendicular to the caustic line, then

$V_{\perp}=\boldsymbol{V} \cdot \boldsymbol{i}=\frac{v_{\mathrm{d}}}{1+z_{\mathrm{d}}} \frac{D_{\mathrm{s}}}{D_{\mathrm{d}}}$

where $v_{\mathrm{d}}=\left|\boldsymbol{v}_{\mathrm{d}} . i\right|$ is the amplitude of the projected peculiar motion of the deflector.

Only one direct measurement of $V=|\boldsymbol{V}|$ is currently available. The measurement is an upper limit on $V$, and consequently on $V_{\perp}$, which was inferred from the analysis of the light curves of the system (Wyithe et al. 1999). So we have decided to adopt a velocity range based on the relation (18) and the relevant observational data. Firstly, one must find plausible values of $v_{\mathrm{d}}$, and with this aim, we analyzed a catalog of galaxy data that is included in the electronic archives of the CDS (Centre de Données astronomiques de Strasbourg). The catalog is a part of the Mark III full catalog of redshifts and distances (Willick et al. 1997), and it contains redshifts in the CMB frame and both forward and inverse Tully-Fisher (TF) distances of 1355 spiral galaxies (Mathewson et al. 1992). As it is well-known, the differences $z_{\mathrm{CMB}}-r_{\mathrm{TF}}$ are the projected peculiar motions of the spirals, i.e., $\boldsymbol{v}_{\mathrm{g}} . \boldsymbol{n}_{\mathrm{g}}$, where $\boldsymbol{n}_{\mathrm{g}}$ are the unit vectors pointing towards the galaxies. In Fig. 4 we can see two very similar histograms showing two distributions of $v_{\mathrm{g}}=\left|\boldsymbol{v}_{\mathrm{g}} \cdot \boldsymbol{n}_{\mathrm{g}}\right|$. The darker line represents the results from the inverse TF distances $\left(r_{\mathrm{ITF}}\right)$, while the other line traces the behaviour from the forward TF distances $\left(r_{\mathrm{FTF}}\right)$. Inhomogeneous Malmquist bias-corrected distances $r_{\mathrm{FTF}}$ are quoted in the catalog, which were computed using the density field obtained through the IRAS $1.2 \mathrm{Jy}$ redshift survey. From the distributions in Fig. 4, we concluded that the mean value is $\left\langle v_{\mathrm{g}}\right\rangle=$ $663 \mathrm{~km} \mathrm{~s}^{-1}$. This average will be within the interval of allowed values of $v_{\mathrm{d}}$. As a lower limit we took $100 \mathrm{~km} \mathrm{~s}^{-1}$, i.e., $v_{\mathrm{d}} \geq 100 \mathrm{~km} \mathrm{~s}^{-1}$, in agreement with the observed distributions $\left[P\left(v_{\mathrm{g}} \leq 50 \mathrm{~km} \mathrm{~s}^{-1}\right) \leq 6 \%\right]$ and discarding a situation in which both $v_{\mathrm{o}}=\left|\boldsymbol{v}_{\mathrm{o}} . i\right|$ and $v_{\mathrm{d}}$ have a value $\leq 50 \mathrm{~km} \mathrm{~s}^{-1}$. On the other hand, the distributions $P\left(v_{\mathrm{g}}\right)$ were obtained with a sample including field spirals and cluster spirals, and it is evident that amplitudes of the projected peculiar velocities in excess of $1000 \mathrm{~km} \mathrm{~s}^{-1}$ cannot be rejected. However, the lens galaxy is not placed inside a rich cluster or close to a group of clusters, and thus, one may assume an upper limit of $1000 \mathrm{~km} \mathrm{~s}^{-1}$, i.e., $v_{\mathrm{d}} \leq 1000 \mathrm{~km} \mathrm{~s}^{-1}$. To derive a reasonable range for $V_{\perp}$, the second basic ingredient is the factor $D_{\mathrm{s}} /\left(1+z_{\mathrm{d}}\right) D_{\mathrm{d}}$. This factor depends on the redshifts $\left(z_{\mathrm{d}}\right.$ and $\left.z_{\mathrm{s}}\right)$ and the matter/energy content of the universe (cosmological parameters). In this paper we

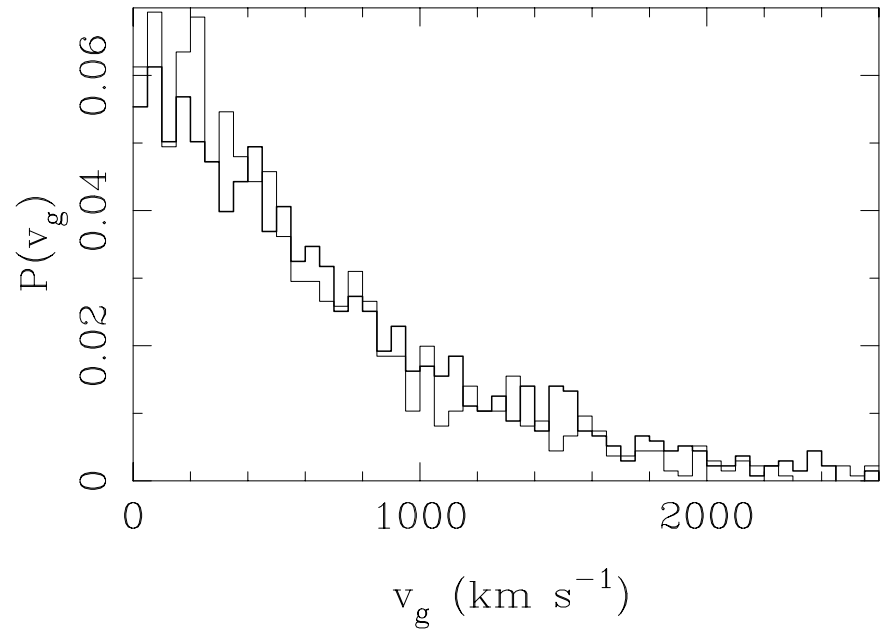

Fig. 4. Probability distributions of the amplitudes of the projected peculiar motions of 1355 nearby spiral galaxies (see Mathewson et al. 1992). The darker line corresponds to the results from the inverse Tully-Fisher distances, and the other line represents the results from the forward Tully-Fisher distances, which were corrected for an inhomogeneous Malmquist bias.

consider the two standard flat cosmologies: (1) $\Omega_{0}=1, \lambda_{0}=0$, and (2) $\Omega_{0}=0.3, \lambda_{0}=0.7$. As usual, $\Omega_{0}$ is the present density parameter and $\lambda_{0}$ is the present reduced cosmological constant. So, each plausible value of $v_{\mathrm{d}}$ leads to two solutions for $V_{\perp}$. Taking into account the whole range $100 \leq v_{\mathrm{d}}\left(\mathrm{km} \mathrm{s}^{-1}\right) \leq 1000$, the characteristic redshifts of the system, and the two standard cosmologies, it is found that $765 \leq V_{\perp}\left(\mathrm{km} \mathrm{s}^{-1}\right) \leq 10548$.

\subsection{Results}

Our results $(1 \sigma$ measurements of $M$ and $\mathrm{d} M / \mathrm{d} t$ for two cosmologies and different values of $v_{\mathrm{d}}$ ) are presented in Table 2. We tested four representative values of $v_{\mathrm{d}}$ (see Sect. 3.2). The black hole mass is reasonably well constrained. For a universe with a zero cosmological constant and a very small projected motion of the deflector, the microlensing data suggest the existence of a central black hole having a mass of $\approx 3.3 \times 10^{7} M_{\odot}$. The most massive black hole is inferred for a universe with a nonzero cosmological constant $\left(\lambda_{0}=0.7\right)$ and a very large projected velocity of the deflector. In that case, $M \approx 4.6 \times 10^{8} M_{\odot}$. Apart from these "individual" estimates, the main result is the global range for $M$. We concluded that the dark mass in the heart of the quasar should be larger than $10^{7} M_{\odot}$ and smaller than $6 \times 10^{8} M_{\odot}$.

With regard to the mass accretion rate, the situation is quite intricate. Firstly, we cannot obtain individual lower limits, since all the $1 \sigma$ estimates are consistent with $\mathrm{d} M / \mathrm{d} t=0$. Therefore, only typical values and upper limits are showed in Table 2. The fact that the $1 \sigma$ error bars on the accretion rate turn out to be compatible with zero is related to the (dis)abilities of the new technique. We fitted the parameters $C$ and $D$ (together with $A_{v}, B_{v}$, and $t_{0}$ ), and used Eqs. (14)-(15) to estimate the physical properties of the central engine: $M$ and $\mathrm{d} M / \mathrm{d} t$. Considering the results in Table $1, f C$ is determined with a relative error of about 40 per cent, while the relative error in $D$ 
Table 2. Black hole mass and mass accretion rate in QSO 2237+0305.

\begin{tabular}{cccc}
\hline \hline Cosmology & $v_{\mathrm{d}}\left(\mathrm{km} \mathrm{s}^{-1}\right)$ & $M\left(10^{8} M_{\odot}\right)$ & $\mathrm{d} M / \mathrm{d} t\left(M_{\odot} \mathrm{yr}^{-1}\right)$ \\
\hline$\Omega_{0}=1, \lambda_{0}=0$ & 100 & $0.33_{-0.19}^{+0.09}$ & $1.5(<3.9)$ \\
& 300 & $1.00_{-0.56}^{+0.26}$ & $13.2(<34.8)$ \\
& 663 & $2.21_{-1.25}^{+0.58}$ & $64.3(<170.0)$ \\
$\Omega_{0}=0.3, \lambda_{0}=0.7$ & 1000 & $3.33_{-1.88}^{+0.87}$ & $146.2(<386.7)$ \\
& 100 & $0.46_{-0.26}^{+0.12}$ & $2.8(<7.3)$ \\
& 300 & $1.38_{-0.78}^{+0.36}$ & $25.0(<66.1)$ \\
& 663 & $3.04_{-1.72}^{+0.79}$ & $122.1(<322.8)$ \\
& 1000 & $4.59_{-2.59}^{+1.20}$ & $277.7(<734.4)$ \\
\hline
\end{tabular}

is of about 45 per cent. If we take a given value of the cosmological parameters and $v_{d}$, then the relative error in $M$ will be of about 40 per cent, i.e., similar to the relative error in $f C$ (see Eq. (14) and the results on $M$ in Table 2). However, unfortunately, Eq. (15) indicates us that $\mathrm{d} M / \mathrm{d} t$ depends on both $(f C)^{2}$ and $D^{4}$. Due to this fact, the accuracy in the determination of $\mathrm{d} M / \mathrm{d} t$ is very poor, or in other words, the relative error in $\mathrm{d} M / \mathrm{d} t$ is greater than 100 per cent.

Secondly, a realistic scenario should incorporate the electron-scattering opacity, which could play an important role at inner regions of the accretion disk (Shakura \& Sunyaev 1973). The local radiation energy flux is determined by the gravitational energy release $Q_{\text {grav }}=$ $(3 / 8 \pi)\left(G M / r^{3}\right)(\mathrm{d} M / \mathrm{d} t)\left[1-\left(r_{\text {in }} / r\right)^{1 / 2}\right]$. In the outer regions, where the free-free processes give the main contribution to the opacity, a Planck spectrum is formed. The radiation energy flux must be $Q_{\text {rad }}=\sigma T_{s}^{4}$, and from $Q_{\text {rad }}=Q_{\text {grav }}$ we obtain the standard thermal law. In the inner regions of the disk, the electron-photon scattering presumably plays a role in the opacity, so these regions radiate less efficiently than a blackbody: $I_{v_{s}}\left[T_{\mathrm{s}}(r)\right]=\varepsilon_{\text {in }}\left[v_{\mathrm{s}}, T_{\mathrm{s}}(r)\right] B_{v_{\mathrm{s}}}\left[T_{\mathrm{s}}(r)\right]$ with $\varepsilon_{\text {in }}\left[v_{\mathrm{s}}, T_{\mathrm{s}}(r)\right]<1$. If one defines a constant factor $\varepsilon_{\text {in }}<1$ to be the emissivity relative to a blackbody, then the corresponding radiation energy flux will be $Q_{\text {rad }}=\varepsilon_{\text {in }} \sigma T_{\mathrm{s}}^{4}$. At small radii the real disk could be hotter than the standard disk without electron-photon scattering, i.e., $T_{\mathrm{s}}(r)=\left[(3 / 8 \pi)\left(G M / \sigma r^{3}\right) \varepsilon_{\text {in }}^{-1}(\mathrm{~d} M / \mathrm{d} t)\left(1-\sqrt{r_{\text {in }} / r}\right)\right]^{1 / 4}$. Therefore we can consider two simple pictures: a global blackbody spectrum ( $\varepsilon=1$ along all the disk), i.e., the standard one, and a global greybody spectrum $(\varepsilon<1$ along all the disk). This last simplification was suggested by Rauch \& Blandford (1991), who introduced the greybody spectra in microlensing studies. When a generalized model is considered $(\varepsilon \leq 1)$, we must re-interpret the results of $\mathrm{d} M / \mathrm{d} t$ as measurements of $\varepsilon^{-1}(\mathrm{~d} M / \mathrm{d} t)$. In this way, the typical values of $\mathrm{d} M / \mathrm{d} t$ vary in the interval (1-300) $\varepsilon M_{\odot} \mathrm{yr}^{-1}$. For $\varepsilon=1$, we derive a typical range of $1-300 M_{\odot} \mathrm{yr}^{-1}$, while for $\varepsilon=0.1$, the typical mass accretion rates are $0.1-30 M_{\odot} \mathrm{yr}^{-1}$.

\section{Conclusions and discussion}

We showed the time evolution of a microlensing HME caused by a generic Newtonian geometrically-thin and optically-thick standard accretion disk crossing a generic caustic straight line. Given a lensed QSO, in order to estimate the mass of its central black hole and the accretion rate, we can compare the theoretical law with a gravitational microlensing HME observed in some image of the far source. This technique was applied to the gravitational mirage QSO 2237+0305, whose brightest image experienced an important change in flux during the 1999-2000 seasons (Woźniak et al. 2000). The peak of the dramatic variation in Q2237+0305A (Alcalde et al. 2002) led to very interesting information on the black hole in the core of the source and an interval of typical values for the mass accretion rate. The main source of uncertainty comes from our lack of knowledge about the peculiar motion of the lens galaxy in the direction perpendicular to the caustic line $\left(v_{\mathrm{d}}\right)$. To accurately determine the typical flow of matter, it is also necessary to know the physical processes involved in the emissivity of the disk. Although the standard scenario does not incorporate the electron-photon scattering, in general, it could strongly distort the standard Planck spectrum at the innermost layers of the disk (e.g., Shakura \& Sunyaev 1973; Malkan 1983).

From $1 \sigma$ estimates of the relevant parameters in the theoretical microlensing curve, we inferred $1 \sigma$ measurements of the black hole mass and the accretion rate for a set of $v_{\mathrm{d}}$ values, which represents the whole range of reasonable choices (see a detailed discussion in Sect. 3.2). Taking into account all the individual estimates in Table 2, one obtains that QSO 2237+0305 contains a massive black hole: $10^{7} M_{\odot}<M<6 \times 10^{8} M_{\odot}$. The information about the mass accretion rate is very much poorer. The main result consists of a typical interval for $\mathrm{d} M / \mathrm{d} t$. This interval is of $(1-300) \varepsilon M_{\odot} \mathrm{yr}^{-1}$, where $\varepsilon=1$ if the free-free processes are dominant (standard source) and $\varepsilon<1$ when the scattering plays a role (e.g., Rauch \& Blandford 1991). In spite of the fact that the range of typical determinations of $\mathrm{d} M / \mathrm{d} t$ is not excessively broad, all the individual $1 \sigma$ measurements are in agreement with no accretion, but a flow of matter of almost $1000 \varepsilon M_{\odot} \mathrm{yr}^{-1}$ cannot be ruled out. On the other hand, one direct constraint on $v_{d}$ was recently reported by Wyithe et al. (1999). This group is involved in a project to interpret the QSO 2237+0305 microlensing light-curves, and as a part of the program, they derived an upper limit of $v_{\mathrm{t}}<500 \mathrm{~km} \mathrm{~s}^{-1}$, where $v_{\mathrm{t}}$ is the galactic transverse velocity $\left(\Omega_{0}=1, \lambda_{0}=0\right)$. Therefore, considering that $v_{\mathrm{d}}<500 \mathrm{~km} \mathrm{~s}^{-1}$ and $v_{\mathrm{d}} \geq 100 \mathrm{~km} \mathrm{~s}^{-1}$, we find a central value of $v_{\mathrm{d}}=300 \mathrm{~km} \mathrm{~s}^{-1}$. For a flat universe with a zero cosmological constant and a projected motion of $v_{\mathrm{d}}=$ $300 \mathrm{~km} \mathrm{~s}^{-1}$, the typical source parameters are: $M=10^{8} M_{\odot}$ and $\mathrm{d} M / \mathrm{d} t \approx 10 \varepsilon M_{\odot} \mathrm{yr}^{-1}$.

Several modeling aspects can be improved. For example, one must include the effects of general relativity. One may also incorporate a new theoretical law in the gallery of models: the time evolution of a microlensing event generated by an accretion disk passing close to a cusp caustic. Efforts in both directions are now in progress, and we will consider these improvements in future studies of new QSO 2237+0305 microlensing peaks. Fortunately, using the present model and monitoring the events that occur in the components of the system, the future prospects are very promising. Thus, from $10 \mathrm{mi}-$ crolensing peaks monitored in ten different optical bands (blue bands are required to avoid the possible contamination by other sources of light as the extrapolated IR power law spectrum, and therefore, the possible perturbation of the chromatic amplitude 
$A_{v}$ which is defined in Eq. (10) for a blackbody emission; see, for example, Malkan 1983), we must be able to noticeably reduce the current uncertainty in the microlensing parameter $D$ : from about 0.3 (see Table 1) to 0.03 . In a similar way, if each peak is recorded at 10 frequencies, the error in each $C$ value will be lowered in a factor of about 3 , i.e., from a mean uncertainty of $\approx 0.1$ day $^{-1}$ to $\approx 0.03 \mathrm{day}^{-1}$. As a result of new detailed monitoring programs, accuracies of $5-10 \%$ in the microlensing parameters $C$ and $D$ can be easily achieved in the next years. If a global dataset is made for each microlensing peak (tracing the time evolution of the spectrum), and it is fitted to a model with six free parameters: $A, B, C, D, t_{0}$, and $\gamma$ $\left(A_{v}=A v^{3+\gamma}, B_{v}=B v^{3+\gamma}\right)$, the accuracy could be even better than a few percent. However, as it was previously remarked, we need a robust estimation of the lens galaxy motion to accurately measure the black hole mass. This "dynamical problem" is the only pitfall to obtain a robust measurement of the amount of dark mass in the centre of QSO 2237+0305. From another point of view, if an independent estimate of $M$ were available, we would deduce the effective quasar velocity $V_{\perp}$ involved in the different microlensing events as well as the value of $\varepsilon^{-1}(\mathrm{~d} M / \mathrm{d} t)$. Through a reasonable collection of $V_{\perp}$ data, one may derive the effective transverse velocity of the quasar $(V)$, which is a basic piece in some microlensing analyses. Up to now we focussed on the parameters $(C, D)$ and the properties of the source, however, the parameters $\left(A_{v}, B_{v}\right)$ are also relevant for other studies. For example, if a multiband monitoring of a microlensing peak is available, we may estimate the ratios $A_{v(i)} / A_{v(j)}$. These ratios will be directly related to the extinction ratios $\epsilon_{v(i)} / \epsilon_{v(j)}$, and consequently, to the global (host galaxy + lens galaxy + Milky Way) extinction law.

Finally, we wish to put the new technique into perspective to determine the physical parameters of a far quasar and the measurements for QSO 2237+0305. Evidence for central black holes in local galaxies comes from small rotating gaseous and stellar disks discovered with the Hubble Space Telescope, VLBI observations of $\mathrm{H}_{2} \mathrm{O}$ masers in Keplerian rotation in some galactic nuclei, and near-infrared measurements of the radial and proper motions of stars in the cluster at the centre of the Galaxy (e.g., Ford et al. 1998). Analyzing these gravitational signatures of massive black holes, the typical values of the black hole masses vary between $\sim 3 \times 10^{6} M_{\odot}$ for the Milky Way (Genzel et al. 1997; Ghez et al. 1998) and $\sim 3 \times 10^{9} M_{\odot}$ for M87 (Ford et al. 1996; Macchetto et al. 1997). The central dark mass can be also measured in far AGNs using two classical methods: analyses of the blue and ultraviolet regions of the rest-frame spectra (e.g., Malkan 1983; Sun \& Malkan 1989) and observations of the time delays between the variations of the continua and the variations of the broad emission lines (e.g., Wandel et al. 1999). The reverberation technique provides an estimate of the size of the broad-line region (BLR) as well as the central mass of a quasar at redshift $z_{\mathrm{QSO}}$. If the line-emitting gas is gravitationally bound to the central black hole, the virial theorem implies that $M \propto R_{\mathrm{BLR}} \sigma^{2}$, where $\sigma$ is the $F W H M$ of the emission profile of the BLR at distance $R_{\mathrm{BLR}}$. Therefore, measuring the lag $\tau$ between an intrinsic event at the central continuum source and the reply (reverberation) at the BLR, one obtains the size $R_{\mathrm{BLR}}=c \tau /\left(1+z_{\mathrm{QSO}}\right)$ and the dark mass $M$ (e.g.,
Vestergaard 2002 and references therein). Apart from the reverberation mapping technique, there is a straightforward method to get the parameters $M$ and $\mathrm{d} M / \mathrm{d} t$. A comparison between the blue-ultraviolet excess flux of a QSO (over the extrapolated infrared power-law spectrum) and the theoretical integrated disk spectrum permits to deduce the two physical quantities (e.g., Malkan 1983). In a pioneering work, Shields (1978) found $M=10^{9} M_{\odot}$ and $\mathrm{d} M / \mathrm{d} t=3 M_{\odot} \mathrm{yr}^{-1}$ for a Newtonian blackbody disk modelling the central engine in 3C 273. To make the fits, Malkan (1983) used a relativistic standard accretion disk. The theoretical model included the effects of general relativity, but the inclination and scattering atmosphere were ignored. For a sample of 6 quasars and assuming nonrotating black holes, the "big blue bumps" can be fitted by spectra of disks around massive $\left(\sim 10^{8} M_{\odot}\right)$ black holes. The author also concluded that the six high-luminosity quasars are emitting energy at approximately their Eddington limits. The effects of disk inclination have been more recently considered by Sun \& Malkan (1989), while the effects of electron scattering were discussed by Wandel \& Petrosian (1988). In this paper we applied an alternative methodology to determine $(M, \mathrm{~d} M / \mathrm{d} t)$ : the monitoring of a lensed quasar when a microlensing HME occurs (e.g., Yonehara et al. 1998; Agol \& Krolik 1999). The method can be seen as a complementary tool to prove the existence of massive dark objects and accretion disks in the centre of far QSOs. Taking as reference values $M=10^{8} M_{\odot}$ and $\mathrm{d} M / \mathrm{d} t \approx 10 \varepsilon M_{\odot} \mathrm{yr}^{-1}$ (see here above), the estimate of the central dark mass in QSO $2237+0305$ is consistent with data of other galaxy nuclei. However, the accretion rate is relatively large for a blackbody disk $(\varepsilon=1)$. As $L / L_{\text {Edd }} \propto(\mathrm{d} M / \mathrm{d} t) / M$, where $L$ is the total disk luminosity and $L_{\mathrm{Edd}}$ is the Eddington luminosity, one obtains $L / L_{\text {Edd }} \sim 3$ for $\varepsilon=1, L / L_{\text {Edd }} \sim 1$ for $\varepsilon=0.3$, and $L / L_{\mathrm{Edd}} \sim 0.3$ for $\varepsilon=0.1$. Therefore, to avoid meaningless results $\left(L>L_{\text {Edd }}\right)$, the electron-photon scattering must play a role.

Acknowledgements. We would like to thank Vyacheslav Shalyapin for his insights on fitting procedures. We also thank Joachim Wambsganss and the anonymous referee for support during the project and useful comments, respectively. The GLITP observations were made with Nordic Optical Telescope (NOT), which is operated on the island of La Palma jointly by Denmark, Finland, Iceland, Norway, and Sweden, in the Spanish Observatorio del Roque de Los Muchachos of the Instituto de Astrofísica de Canarias (IAC). We are grateful to the technical team of the telescope for valuable collaboration during the observational work. This work was supported by the P6/88 project of the IAC, Universidad de Cantabria funds, DGESIC (Spain) grant PB97-0220-C02, and the Spanish Department for Science and Technology grants AYA2000-2111-E and AYA2001-1647-C02.

\section{Appendix A: Approximation to the function $G(q)$}

The function $G(q)$ is defined from Eq. (7) of the main text. For $q>1$, we can rewrite $G(q)$ as

$G(q)=\frac{1}{\sqrt{q}} \int_{-1}^{+1} \frac{\mathrm{d} y}{\sqrt{1-y^{2}}}(1-y / q)^{-1 / 2}$ 
and expand the factor $(1+x)^{-1 / 2}, x=-y / q,-1<x<1$. Then we derive an approximated law

$G(q) \simeq \frac{\pi\left(1155+1680 q^{2}+3072 q^{4}+16384 q^{6}\right)}{16384 q^{13 / 2}}$,

which works very well at $q>2$ (relative deviations less than $0.025 \%$ ). However, the previous expansion does not work so well in the interval $1<q \leq 2$. Taking $u=1 / \sqrt{q-y}$ and $\mathrm{d} v=\mathrm{d} y / \sqrt{1-y^{2}}, G(q)$ will be equal to

$G(q)=\frac{\pi}{2}\left(\frac{1}{\sqrt{q-1}}+\frac{1}{\sqrt{q+1}}\right)+K(q)$,

with

$K(q)=-\frac{1}{2} \int_{-1}^{+1} \mathrm{~d} y \frac{\arcsin y}{(q-y)^{3 / 2}}$.

Expanding the function $\arcsin y$, we can approximate $K(q)$ (and thus, $G(q)$ ) as

$K(q) \simeq \frac{1+2 q}{\sqrt{q+1}}+\frac{1-2 q}{\sqrt{q-1}}+\ldots$

At $q \approx 1$ the first approach (based on the expansion of $\left.(1-y / q)^{-1 / 2}\right)$ underestimates the true behaviour, while the new scheme (based on the expansion of $\arcsin y$ ) overestimates $G(q)$. Due to this fact, at $1<q \leq 2, G(q)$ is fitted to a law incorporating both trends. The final result is

$G(q) \simeq \frac{\sum_{i=0}^{2} a_{i} q^{i}}{q^{5 / 2}}+\frac{\sum_{i=0}^{2} b_{i} q^{i}}{\sqrt{q-1}}+\frac{\sum_{i=0}^{2} c_{i} q^{i}}{\sqrt{q+1}}$,

where $a_{0}=2.89283135146944, a_{1}=0, a_{2}=$ $125.8537652305732, b_{0}=-102.1055508185553, b_{1}=$ $227.8006617807037, \quad b_{2}=-125.694998412877, \quad c_{0}=$ $-202.3343397590727, c_{1}=-93.3006092335309$, and $c_{2}=$ 124.1342775121369. The approximation (A.6) is very accurate (relative deviations $\leq 0.01 \%$ ), even at $q$ values very close to the singularity $(q=1)$.

For $q<1$, it is more difficult to obtain useful approaches to $G(q)$. After some tests, we considered the combination of a smooth law (i.e., $\left.d_{0}+d_{1} q+d_{2} q^{2}+\ldots\right)$ and a trend that is singular at $q=1$ (i.e., $\left.\left(e_{0}+e_{1} q+e_{2} q^{2}+\ldots\right) / \sqrt{1-q}\right)$. At $-1<q<1$, from the combined law we infer a good fit

$G(q) \simeq \sum_{i=0}^{8} \mathrm{~d}_{i} q^{i}+\frac{\sum_{i=0}^{8} e_{i} q^{i}}{\sqrt{1-q}}$,

where $d_{0}=353.7491560566996, d_{1}=-29.22210584958317$, $d_{2}=-285.8452207779682, d_{3}=-296.6766633600129$, $d_{4}=-77.10649562470328, \quad d_{5}=236.0210288747884$, $d_{6}=329.3059620964406, d_{7}=-240.8295381320522$, $d_{8}=18.61151978935351, e_{0}=-351.1273028283772$, $e_{1}=205.3847185910097, e_{2}=315.1612274462573, e_{3}=$ $171.9981477672829, \quad e_{4}=-95.1705826992738, e_{5}=$ $-320.8255310602542, e_{6}=-243.8876547053204, e_{7}=$ 415.2244998079605, and $e_{8}=-96.7574150920885$. The relative deviations are again less than $0.02 \%$. Of course $G(q)=0$ at $q \leq-1$.

\section{References}

Agol, E., \& Krolik, J. 1999, ApJ, 524, 49

Alcalde, D., Mediavilla, E., Moreau, O., et al. 2002, ApJ, 572, 729

Ford, H., Ferrarese, L., Hartig, G., et al. 1996, in Proc. Nobel Symp. 98, Barred Galaxies and Circumnuclear Activity, ed. A. Sandqvist, \& P. Lindblad (Springer-Verlag, Heidelberg), 293

Ford, H., Tsvetanov, Z., Ferrarese, L., \& Jaffe, W. 1998, in The Central Regions of the Galaxy and Galaxies, ed. Y. Sofue (Kluwer, Dordrecht), Proc. IAU Symp., 184, 377

Genzel, R., Eckart, A., Ott, T., \& Eisenhauer, F. 1997, MNRAS, 291, 219

Ghez, A. M., Klein, B. L., Morris, M., \& Becklin, E. E. 1998, ApJ, 509,678

Jaroszyński, M., Wambsganss, J., \& Paczyński, B. 1992, ApJ, 396, L65

Kayser, R., Refsdal, S., \& Stabell, R. 1986, A\&A, 166, 36

Lineweaver, C. H., Tenorio, L., Smoot, G. F., et al. 1996, ApJ, 470, 38

Macchetto, F., Marconi, A., Axon, D. J., et al. 1997, ApJ, 489, 579

Malkan, M. A. 1983, ApJ, 268, 582

Mathewson, D. S., Ford, V. L., \& Buchhorn, M. 1992, ApJS, 81, 413

Mineshige, S., \& Yonehara, A. 1999, PASJ, 51, 497

Nelder, J. A., \& Mead, R. 1965, Computer J., 7, 308

Novikov, I. D., \& Thorne, K. S. 1973, in Black Holes, ed. C. DeWitt, \& B. S. DeWitt (Gordon \& Breach, New York), 343

Page, D. N., \& Thorne, K. S. 1974, ApJ, 191, 499

Rauch, K. P., \& Blandford, R. D. 1991, ApJ, 381, L39

Schneider, P., \& Weiss, A. 1987, A\&A, 171, 49

Shakura, N. I., \& Sunyaev, R. A. 1973, A\&A, 24, 337

Shalyapin, V. N. 2001, AstL, 27, 150

Shalyapin, V. N., Goicoechea, L. J., Alcalde, D., et al. 2002, ApJ, 579, 127

Shields, G. A. 1978, Nature, 272, 706

Sun, W., \& Malkan, M. A. 1989, ApJ, 346, 68

Vestergaard, M. 2002, ApJ, 571, 733

Wandel, A., Peterson, B. M., \& Malkan, M. A. 1999, ApJ, 526, 579

Wandel, A., \& Petrosian, V. 1988, ApJ, 329, L11

Willick, J. A., Courteau, S., Faber, S. M., et al. 1997, ApJS, 109, 333

Woźniak, P. R., Udalski, A., Szymański M., et al. 2000, ApJ, 540, L65

Wyithe, J. S. B., Webster, R. L., \& Turner, E. L. 1999, MNRAS, 309, 261

Yonehara, A. 2001, ApJ, 548, L127

Yonehara, A., Mineshige, S., Fukue, J., Umemura, M., \& Turner, E. L. 1999, A\&A, 343, 41

Yonehara, A., Mineshige, S., Manmoto, T., et al. 1998, ApJ, 501, L41 